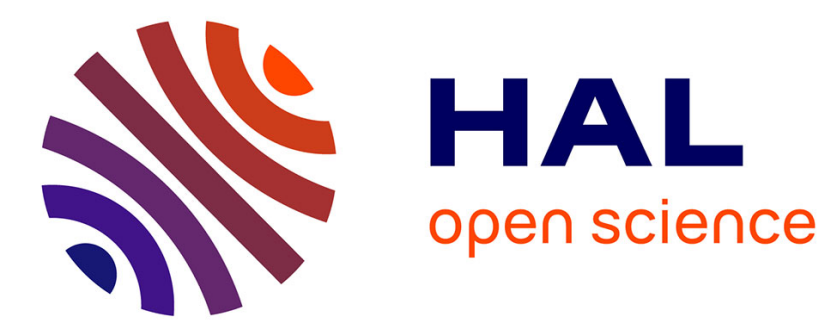

\title{
Tunable bimodal explorations of space from memory-driven deterministic dynamics
}

Maxime Hubert, Stéphane Perrard, Matthieu Labousse, Nicolas Vandewalle, Yves Couder

\section{- To cite this version:}

Maxime Hubert, Stéphane Perrard, Matthieu Labousse, Nicolas Vandewalle, Yves Couder. Tunable bimodal explorations of space from memory-driven deterministic dynamics. Physical Review E , 2019, 100 (3), 10.1103/PhysRevE.100.032201 . hal-02349244

\section{HAL Id: hal-02349244 \\ https://hal.science/hal-02349244}

Submitted on 5 Nov 2019

HAL is a multi-disciplinary open access archive for the deposit and dissemination of scientific research documents, whether they are published or not. The documents may come from teaching and research institutions in France or abroad, or from public or private research centers.
L'archive ouverte pluridisciplinaire HAL, est destinée au dépôt et à la diffusion de documents scientifiques de niveau recherche, publiés ou non, émanant des établissements d'enseignement et de recherche français ou étrangers, des laboratoires publics ou privés. 


\title{
Tunable bimodal explorations of space from memory-driven deterministic dynamics
}

\author{
Maxime Hubert* \\ GRASP, Institute of Physics, Université de Liège, 4000 Liège, Belgium, European Union \\ Stéphane Perrard \\ Laboratoire de Physique de l'ENS, CNRS UMR 8550 ENS and PSL University, 75005 Paris, European Union \\ Matthieu Labousse $\oplus^{\dagger}$ \\ Gulliver, CNRS UMR 7083, ESPCI Paris and PSL University, 75005 Paris, France, European Union \\ Nicolas Vandewalle \\ GRASP, Institute of Physics, Université de Liège, 4000 Liège, Belgium, European Union \\ Yves Couder \\ Matière et Systèmes Complexes, CNRS UMR 7057, Université Paris Diderot, Sorbonne Paris Cité, \\ 75013 Paris, France, European Union
}

(Received 30 July 2018; revised manuscript received 28 March 2019; published 3 September 2019)

\begin{abstract}
We present a wave-memory-driven system that exhibits intermittent switching between two propulsion modes in free space. The model is based on a pointlike particle emitting periodically cylindrical standing waves. Submitted to a force related to the local wave-field gradient, the particle is propelled, while the wave field stores positional information on the particle trajectory. For long memory, the linear motion is unstable and we observe erratic switches between two propulsive modes: linear motion and diffusive motion. We show that the bimodal propulsion and the stochastic aspect of the dynamics at long time are generated by a Shil'nikov chaos. The memory of the system controls the fraction of time spent in each phase. The resulting bimodal dynamics shows analogies with intermittent search strategies usually observed in living systems of much higher complexity.
\end{abstract}

DOI: 10.1103/PhysRevE.100.032201

\section{INTRODUCTION}

At the individual level, random switches between straightline motion and erratic changes in direction has been observed in many living systems such as microscopic bacteria [1], flies [2,3], or macroscopic animal foraging [4]. The theoretical description of animal foraging based on bimodal exploration of space cannot be separated from a fine analysis of the spatial heterogeneities of their environment $[5,6]$. However, in some specific cases, the origin of bimodal motions may be justified using optimal search strategies in the absence of cues [7]. Indeed, without any cues about the target location, bimodal motions can be encountered when exploration and exploitation tasks are not performed simultaneously, for instance, in fishes looking for prey of various sizes [8]. In contrast, when the searcher can perform both tasks simultaneously, Levy flights strategies $[9,10]$ with exponents depending on the target properties are optimal even if their observations and

\footnotetext{
*Present address: PULS Group, Department of Physics and Interdisciplinary Center for Nanostructured Films, Friedrich-AlexanderUniversity Erlangen-Nuremberg, Cauerstr. 3, 91058 Erlangen, Germany, European Union.

${ }^{\dagger}$ matthieu.labousse@espci.psl.eu
}

relevances in the context of animal motions has been raised [11-13].

The theoretical description of these individual trajectories usually involves a pinch of stochasticity as can be seen from the pioneering work of Pearson [14] up to the numerous recent theoretical analysis using persistent random walks $[15,16]$. Individual trajectories of erratic aspects can also be obtained from purely deterministic rules, in which stochasticity becomes an emergent behavior $[17,18]$. Emergent complexity from simple deterministic model rules would provide a robust artificial implementation of bimodal explorations in which the statistics of both phases of motion would not be preset in an arbitrary manner. Ideally, few tunable parameters would control the proportion of time spent in each phase. To achieve this goal, in this article, we focus on the interconnected dynamics of a particle and a scalar field. The model we propose uses the concepts of both chaos theory and time-delayed systems. Indeed, the particle is coupled in a deterministic and nonlinear way to the scalar field while the scalar field stores informations about the particle position, acting as a readable memory.

The design of our model finds its origin in the dynamics of walking droplets on a vertically vibrated bath $[19,20]$. A silicone droplet compelled to bounce on a vertically vibrated liquid surface generates monochromatic cylindrical standing waves by its successive impacts, thanks to the immediate 
vicinity of the Faraday instability [21]. In return, the waves propel the droplet along the surface. The waves are slowly damped in time, with the temporal decay controlled by the bath acceleration [22]. Besides this particular experimental implementation, it is an example of physical trajectory encoding information in a surrounding wave medium [22,23]. The center of each circular wave pattern is located at the exact previous position of the walker, such that a positional information is periodically stored in an oscillating wave field $[22,24,25]$. Deposition of information along a path is also encountered in living systems such as ants [26] and may lead to bioinspired algorithmic principles [27]. Here the wave persistence defines a memory time during which the positional information is stored and can be read or erased, similarly to a Turing machine [23]. Besides wave-particle-inspired dynamics $[19,20,24,25,28-42]$, walking droplets exhibit cascades of bifurcation to chaos in Coriolis and Coulomb force field [43] as well as intermittency in harmonic potential [36,43-48]. Nonsteady propulsions have been reported in asynchronous bouncing modes [49,50] and speed limit cycle and chaotic behavior for the free particle [51] have been investigated for synchronous bouncing modes. In short, we have in our hand a particle (the droplet) coupled nonlinearly to a scalar field (the height of the interface) where the memory drives and tunes the dynamics of both subsystems.

In this article we leverage this concept of wave memory to implement three modes of motion at the single-particle level in the same model: ballistic, diffusive, and intermittent motions. The article is organized as follows. In Sec. II, we describe the model and its implementation. In Sec. III, we analyze the evolution of the trajectories as the memory parameter of the system is increased. We show that above a critical memory parameter the system exhibits a transition from ballistic path to a bimodal mode of motion, namely an intermittent succession of ballistic and erratic motion. Then we rationalize in Sec. IV the chaotic nature of the intermittency and show that a Shil'nikov mechanism triggers the switch between the two modes of motion. Finally, in Section V, we analyze the statistical properties of the long-term dynamics in the light of the local intermittent behavior.

\section{MODEL}

The experiment-inspired numerical model is implemented as follow. The iterative dynamics consists in the equation of motion of a particle at the position $\vec{r}_{k}$ at the $k$ th bounce and its associated self-built wave field $\zeta(\vec{r}, t)$. The motion is decomposed in two phases of respective duration $t_{1}$ and $t_{2}$ such that $t_{1}+t_{2}=T$ is the wave period, which will be used as the unit of time. Phase 1 corresponds to a free flight motion, in which the particle follows a planar motion above the wave field at constant horizontal velocity. Phase 2 corresponds to the contact with the surface, which yields both the interaction with the waves and the energy dissipation. The particle slides on the surface for a duration $t_{2}$ with an exponentially decaying speed before taking off again. At the peculiar instant between phase 1 and phase 2, the particle gets a kick of momentum proportional to the local slope of the wave field. The wave field is updated simultaneously. A new standing cylindrical wave is added, centered at the current position of the particle.
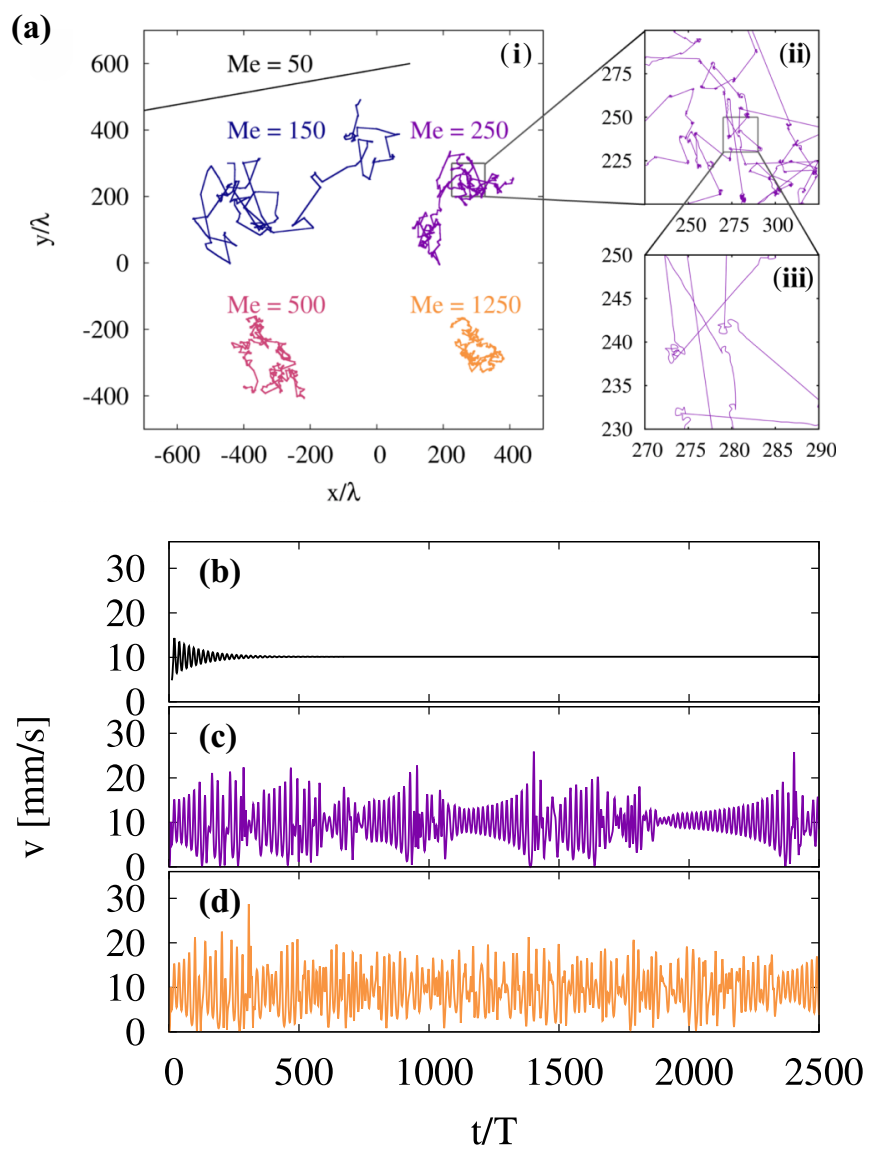

FIG. 1. Evolution of the wave-memory-driven particle dynamics with increasing memory. (a) Particle trajectories obtained for increasing values of $\mathrm{Me}=50,150,250,500,1250$. They correspond to the same simulation time. [(i)-(iii)] Zoom into one trajectory shows evidence of a bimodal dynamics. Straight lines alternate with erratic motion. [(b)-(d)] Particle speed as a function of time for increasing memory parameters (b) $\mathrm{Me}=50$, (c) $\mathrm{Me}=250$, and (d) $\mathrm{Me}=1250$. Above $\mathrm{Me}=150$, the dynamics is unstable. The duration of the laminar phases decrease with Me.

The total wave field $\zeta(\vec{r}, t)$ after the $N$ th bounce at time $t_{N}$ is written as

$$
\zeta\left(\vec{r}, t_{N}\right)=\zeta_{0} \sum_{n=1}^{N} J_{0}\left(\frac{2 \pi}{\lambda}\left|\vec{r}-\vec{r}_{n}\right|\right) e^{-\frac{\left|\vec{r}-\vec{r}_{n}\right|}{\delta}} e^{-\frac{N-n}{M c}},
$$

where $\zeta_{0}$ accounts for the amplitude of one standing cylindrical Bessel wave $J_{0}$ and $\lambda$ is the wavelength. In the inspiring experiments $\lambda \simeq 5 \mathrm{~mm}$, we chose here the wavelength $\lambda$ as the unit of length. The memory parameter Me sets the effective number of active wave sources, and $\delta=2.5 \lambda$ accounts for the spatial attenuation of viscous dissipation [22] (see the Appendix for the numerical values and for a detailed description of the algorithm). The control parameter Me is expected to play a key role in the dynamics.

\section{TRAJECTORIES FOR INCREASING MEMORY}

The particle trajectories for increasing memory parameter Me are shown in Fig. 1(a) (see also the supplemental movies SM1 and SM2 [52]). They are obtained from the 

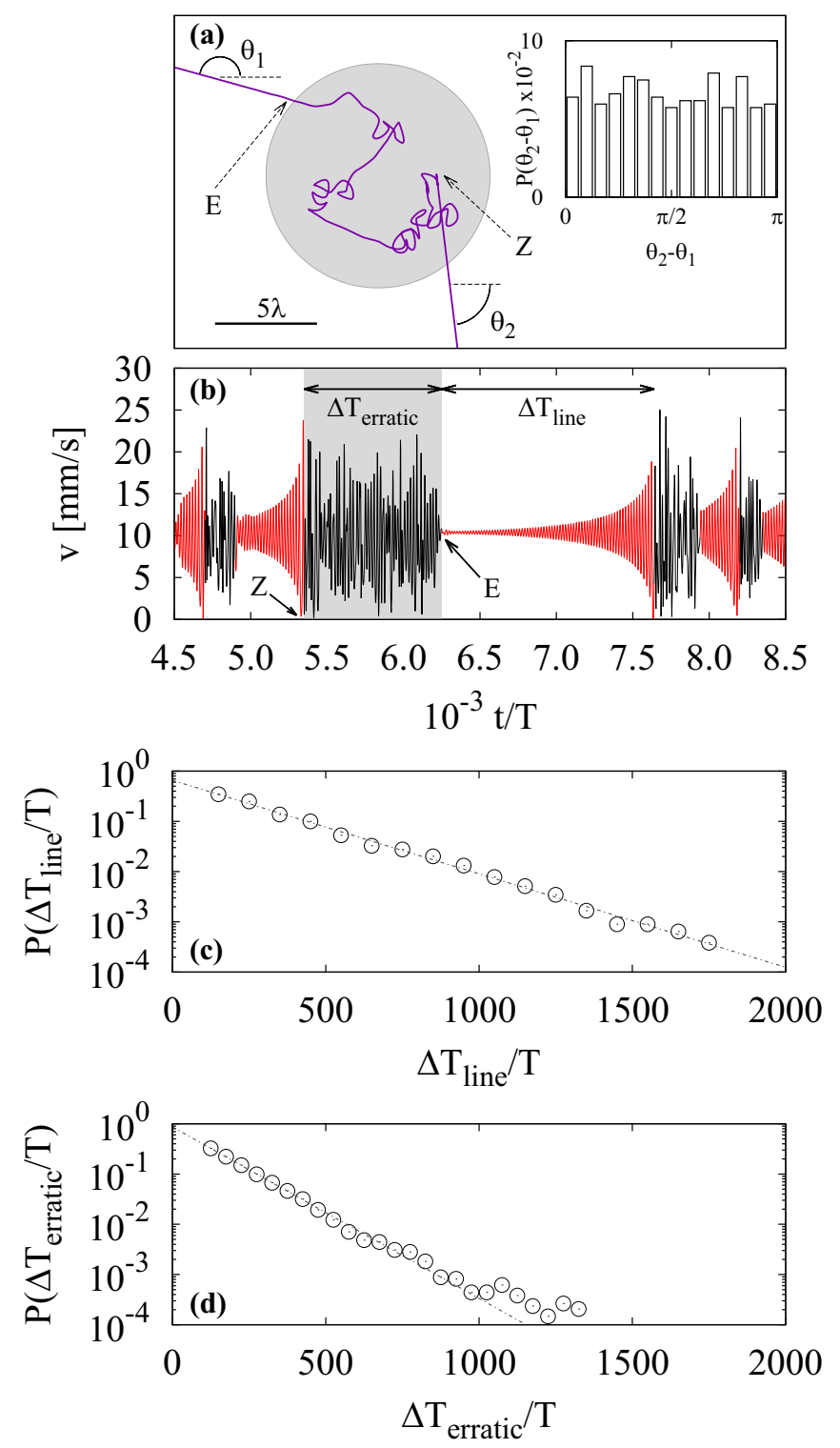

FIG. 2. Description of the bimodal dynamics. (a) Zoom on the trajectory during one erratic phase. Inset: Histogram of $\theta_{2}-\theta_{1}$ showing a uniform distribution. (b) Particle speed as a function of time. The gray zone is an eye guide which corresponds to the laminar phase shown in Fig. 2(a). Erratic motion corresponds to erratic fluctuation of speed (chaotic phase) while linear motions correspond to slow diverging oscillation of speed (laminar phase). (c) [respectively, (d)] Probability distribution (lin-log) of the time spent in linear (respectively, erratic) phase for $\mathrm{Me}=250$.

same initial conditions and a simulation time of $\Delta T_{\text {simu }}=$ $2.5 \times 10^{5} \mathrm{~T}$. For $\mathrm{Me}=50$, the particle moves along a straight line, as described in previous works [24]. This linear motion results from a stable balance between propulsion from the wave field and dissipation by waves emission and viscous friction [28]. Starting from $\mathrm{Me} \simeq 150$, straight-line motions are interspersed by sudden changes of direction. Successive zooms in Fig. 1(a) reveal erratic changes of direction on a scale equal or shorter than the wavelength, alternating with periods of linear motion. For larger Me the length of linear trajectories shorten on average. The memory parameter affects the switching rates between the two modes of space exploration. The switch between linear and erratic phases finds a signature in the particle speed. Figures 1(b)-1(d) present the temporal evolution of the velocity modulus $V$ for increasing memory corresponding to the trajectories shown in Fig. 1(a). For Me = 50 [Fig. 1(b)], transient speed oscillations decrease exponentially in time, and the dynamics converges toward an uniform linear motion. Note that a similar behavior has been observed both experimentally and numerically by Bacot et al. [51]. For $\mathrm{Me}=250$ [Fig. 1(c)], the temporal signal of the particle speed is a succession of oscillations of slowly increasing amplitude and more complex excursions of short duration. On a longer timescale, we observe intermittent switches between slow diverging speed oscillations (laminar phase) followed by erratic motion (chaotic phase). For longer memory parameter, the laminar phases shorten while chaotic phases become predominant [Fig. 1(d)].

The relation between the trajectories and the speed is described in Fig. 2. Figure 2(a) zooms on a erratic phase between two linear parts of the dynamics at $\mathrm{Me}=250$. Figure 2(b) shows the associated speed oscillations. Linear motion coincides with speed oscillations, while erratic trajectories coincides with chaotic speed fluctuations. We use a local radius of curvature of the trajectory greater than $\lambda$ for at least 100 bounces as a criterion for defining a straight line. Statistical results presented on this manuscript do not depend on the criterion choice. Starting the description from the beginning of a laminar phase, the divergence of speed oscillations leads to vanishing velocity [zero (Z) point in Figs. 2(a) and 2(b)]. This specific moment corresponds to a sharp change of direction in the particle trajectory. At Z, the particle hits the surface wave field with a positive slope, which triggers a back motion and initiates the transition from the linear phase to the erratic phase. Then the particle navigates erratically in a confined region of space during a period $\Delta T_{\text {erratic }}$. The complex trajectory in the erratic phase is attributed to the dynamical trap of the particle created by the wave-field structure (see supplemental movie 2 [52]). After a certain period of time the erratic phase ceases and the particle enters again in a phase of linear motion of duration $\Delta T_{\text {line }}$ [E point in Figs. 2(a) and 2(b)]. The spatial extent of the trajectory in the erratic phase is of the order of 10 wavelengths as emphasized in gray in Fig. 2(a). The histogram inset of Fig. 2(a) reveals that the erratic phases perform a statistically isotropic reorientation of the trajectory. The duration of linear motions follows an exponential distribution as evidenced in Fig. 2(c). As the memory parameter is increased, the distribution of time in the laminar phase remains exponential and the average time decreases. Figure 2(d) gives the distribution of time spent in the erratic phase for the same memory parameter as in Fig. 2(c). An exponential distribution is also observed.

\section{CHARACTERIZATION OF THE CHAOTIC DYNAMICS}

To analyze further the chaotic nature of the dynamics, we decompose the wave field using Graf's theorem into the comoving Frenet basis centered at the position of the particle. Graf's addition theorem states that $J_{0}\left(2 \pi\left|\vec{r}-\vec{r}_{n}\right| / \lambda\right)$ can be decomposed onto a sum of eigenmodes $J_{p}(2 \pi|\vec{r}| / \lambda)$ and $J_{p}\left(2 \pi\left|\vec{r}_{n}\right| / \lambda\right)$ considering the angular difference $\left(\theta-\theta_{n}\right)$ 
between the two vectors [53]. The wave field then writes

$$
\begin{gathered}
\zeta\left(\vec{r}, t_{N}\right)=\zeta_{0} \sum_{n=1}^{N} \sum_{p=-\infty}^{\infty} J_{p}\left(\frac{2 \pi}{\lambda}|\vec{r}|\right) \\
\times J_{p}\left(\frac{2 \pi}{\lambda}\left|\vec{r}_{n}\right|\right) e^{i p\left(\theta-\theta_{n}\right)} e^{-\frac{t_{N}-t_{n}}{\mathrm{Me} T}} \\
=\sum_{p=-\infty}^{\infty} \underbrace{\zeta_{0}\left[\sum_{n=1}^{N} J_{p}\left(\frac{2 \pi}{\lambda}\left|\vec{r}_{n}\right|\right) e^{-i p \theta_{n}} e^{-\frac{t_{N}-t_{n}}{\mathrm{Me} T}}\right]}_{C_{p}} J_{p}\left(\frac{2 \pi}{\lambda}|\vec{r}|\right) e^{i p \theta}
\end{gathered}
$$

where the factor $C_{p}$ corresponds to the amplitude of each eigenmode. The walker position in the Frenet frame is given by $|\vec{r}|=0$ and $\theta=0$. Using the asymptotic behavior of the Bessel function for small argument $J_{p}\left(k_{F}|\vec{r}|\right)=$ $\frac{1}{\Gamma(p+1)}\left(\frac{\pi|\vec{r}|}{\lambda}\right)^{p}+O\left(r^{p+1}\right)$, we obtain

$$
\begin{aligned}
\left.\frac{\partial \zeta}{\partial r}\right|_{\vec{r}=\overrightarrow{0}} & =\frac{\pi\left(C_{1}-C_{-1}\right)}{\lambda}, \\
& =\frac{2 \pi \operatorname{Re}\left(C_{1}\right)}{\lambda}, \\
\left.\frac{1}{|\vec{r}|} \frac{\partial \zeta}{\partial \theta}\right|_{\vec{r}=\overrightarrow{0}} & =\frac{i \pi\left(C_{1}+C_{-1}\right)}{\lambda}, \\
& =-\frac{2 \pi \operatorname{Im}\left(C_{1}\right)}{\lambda} .
\end{aligned}
$$

As a conclusion, the knowledge of the wave force acting on the walker can be completely reduced to the single complexvalued scalar quantity $C_{1}$, which is the amplitude of the first Frenet eigenmode. A subset of the flow for $\mathrm{Me}=250$ is plotted in Fig. 3(a), in the three-dimensional phase space $\left[V, \operatorname{Re}\left(C_{1}\right) / \zeta_{0}, \operatorname{Im}\left(C_{1}\right) / \zeta_{0}\right]$. We observe a converging flow along the axis $\operatorname{Im}\left(C_{1}\right) / \zeta_{0}$ followed by a diverging spiral in the plane $\left[V, \operatorname{Re}\left(C_{1}\right) / \zeta_{0}\right]$. This type of trajectory in phase space is encountered in Shil'nikov-type chaos in which the flow converges toward a saddle point before diverging by spiralling outward in the supplementary plane. To evidence the nature of this chaos, we compute the saddle index $v=$ $t_{I} / t_{D}$ [54] which measures the ratio between the reinjection timescale $t_{I}$ and the timescale $t_{D}$ of the diverging flow. They are measured from the temporal evolution of $\operatorname{Im}\left(C_{1}\right) / \zeta_{0}$ and its quadrature $\operatorname{Re}\left(C_{1}-\left\langle C_{1}\right\rangle\right) / \zeta_{0}$ in the vicinity of the saddle [see Figs. 3(b) and 3(c)]. We find $v=0.05$, which is much smaller than 1, a necessary condition for the existence of a Shil'nikov chaos [54]. Shil'nikov chaos has been observed in several contexts from the Belousov-Zhabotinsky reaction [55], electrode dissolution [56,57], and Chua oscillator [58] to $\mathrm{CO}_{2}$ laser $[59,60]$. It is encountered when a homoclinic cycle interacts with a subcritical Hopf bifurcation [61]. Exponential distribution of the laminar phase have been demonstrated in the context of type-II intermittency [62]. We find that Shil'nikov chaos exhibits here the same feature.
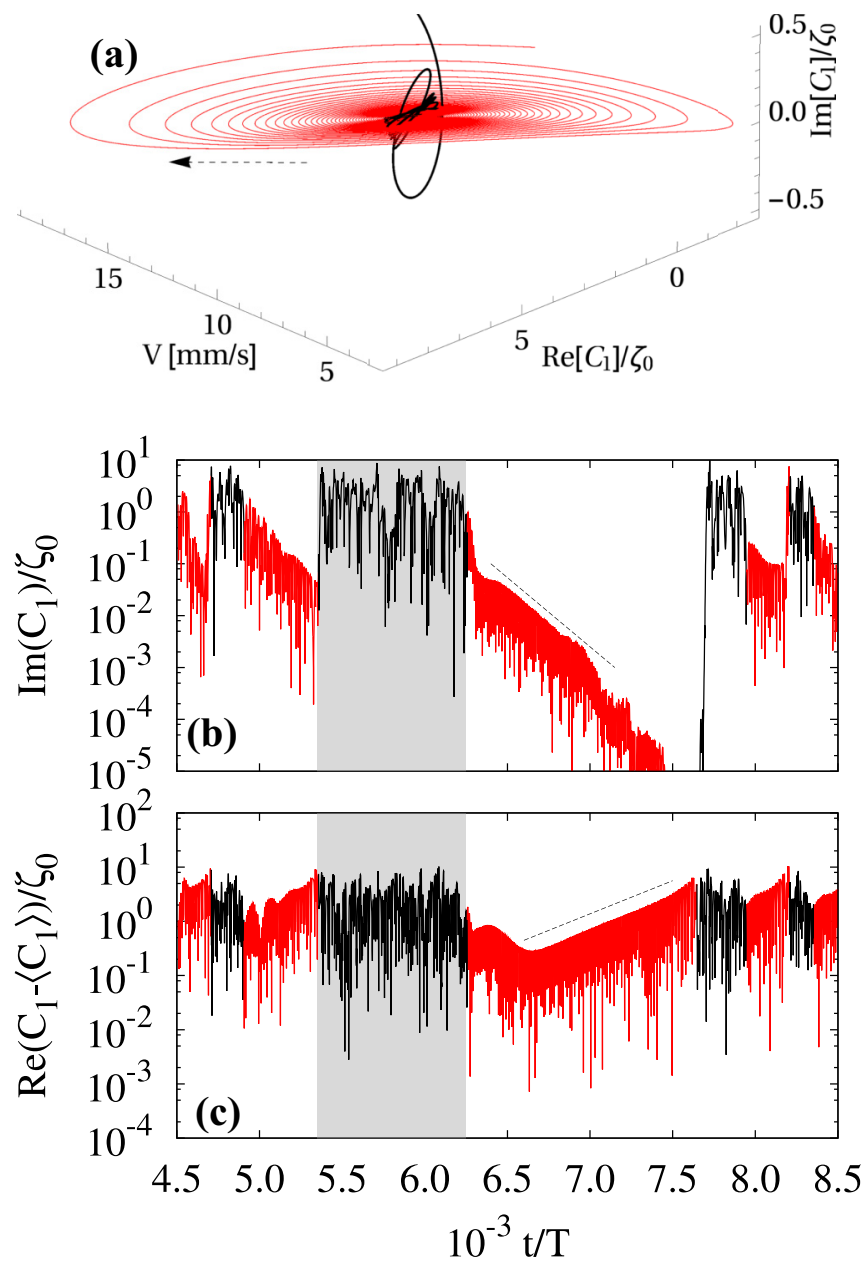

FIG. 3. Characterization of the instability. (a) Three-dimensional representation in the $\left[V, \operatorname{Re}\left(C_{1}\right) / \zeta_{0}, \operatorname{Im}\left(C_{1}\right) / \zeta_{0}\right]$ space for a trajectory at $\mathrm{Me}=250$ [vicinity of the gray part Figs. 2(b) and 2(c)]. The slow outward spiraling flow of the laminar phase alternates with fast out-of-phase inward injection of the erratic phase, a signature of a Shil'nikov-type chaos. [(b) and (c)] Time series of $\operatorname{Im}\left(C_{1}\right) / \zeta_{0}$ and its centered quadrature $\operatorname{Re}\left(C_{1}-\left\langle C_{1}\right\rangle\right) / \zeta_{0}$ in lin-log scale. Dashed lines: Evidence of exponential evolution and the convergence $t_{I}$ and divergence $t_{D}$ time. The color code is as in Figs. 2(b) and 2(c).

\section{STATISTICAL PROPERTIES OF THE LONG-TERM DYNAMICS}

Finally, we analyze the statistical properties of the longterm dynamics by measuring the normalized mean-squared displacement (MSD) of the particle $\left\langle\left|\mathbf{r}(t)-\mathbf{r}_{0}\right|^{2}\right\rangle$, where $\langle$. represents the ensemble average and $t$ is the elapsed time since the position $\mathbf{r}_{0}$. Figure 4(a) shows the MSD as a function of the time $t$ elapsed for increasing memory parameters. For $\mathrm{Me}=$ 100 , the MSD scales as $t^{2}$, as expected for a ballistic motion. For larger memory, the dynamics exhibit three regimes. At short time $t / T<10$, a MSD typical of ballistic motion is recovered. At intermediate timescale $10<t / T<10^{3}$, we observe superdiffusive motion, in which the local MSD exponent $\alpha$ defines as $\alpha=d\left(\log \left(\left\langle\left|\mathbf{r}(t)-\mathbf{r}_{0}\right|^{2}\right\rangle\right) / d t\right.$ lies in the range between 1 and 2. In order to link the MSD with the distribution of time spent in the laminar and chaotic phases, we introduce the autocorrelation function in time of the particle 

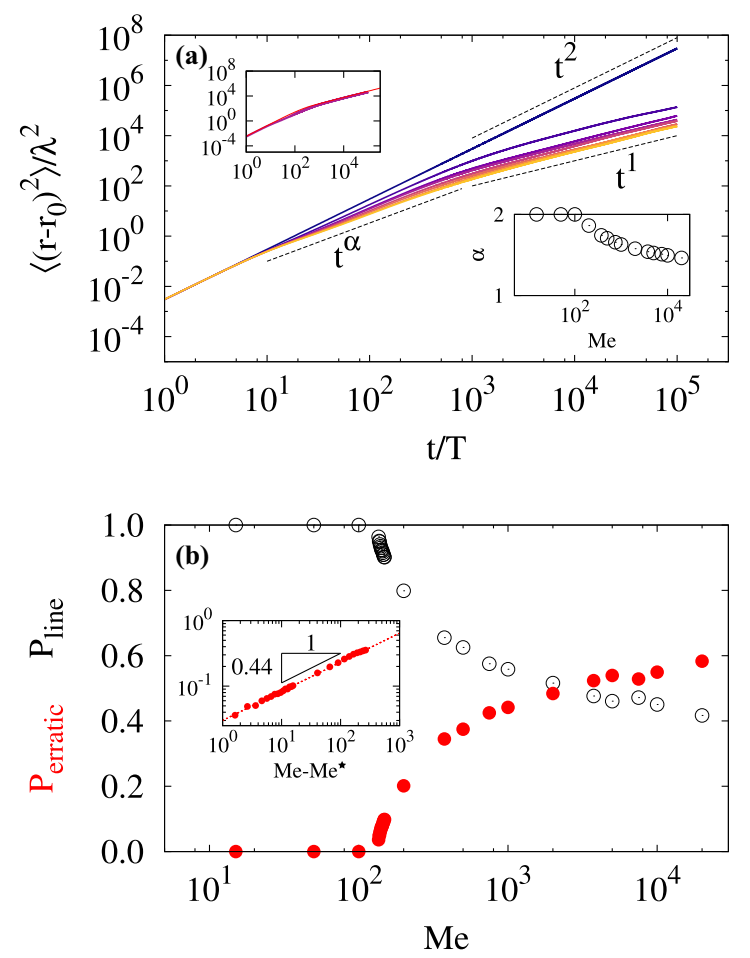

FIG. 4. Statistical properties of long-time dynamics (a) MSD for increasing values of the memory parameter Me (from blue to yellow: 100, 200, 375, 500, 1000, 2000, 5000, 10 ${ }^{4}$ ) as a function of the time $t$. Three regions can be distinguished: ballistic $\left(\frac{t}{T}<10\right)$, superdiffusive $\left(10<\frac{t}{T}<10^{3}\right)$, and diffusive $\left(\frac{t}{T}>10^{3}\right)$. Inset (top); Fit of the MSD using Eq. (11) in the case Me $=500$. Inset (bottom): Evolution of $\alpha$ with $\mathrm{Me}$ in lin-log scale. (b) Measure of $P_{\text {erratic }}$ (respectively, $P_{\text {line }}$ ) the proportion of time spent in the chaotic phase (black circle) [respectively, in the laminar phase (red circle)]. Inset: $P_{\text {erratic }}$ presents a scaling law $P_{\text {erratic }} \sim\left(\mathrm{Me}-\mathrm{Me}^{\star}\right)^{0.44}$.

velocity $\mathbf{v}[63]$ :

$$
C(t)=\langle\mathbf{v}(t) \mathbf{v}(0)\rangle .
$$

The MSD $\left\langle\left|\mathbf{r}(t)-\mathbf{r}_{0}\right|^{2}\right\rangle$ can then be written as:

$$
\frac{d}{d t}\left\langle\left|\mathbf{r}(t)-\mathbf{r}_{0}\right|^{2}\right\rangle=2 \int_{0}^{t} C(u) d u .
$$

The temporal correlation function $C$ can be modelled using the statistical description of the time spent in the linear and the erratic phases. Considering two instants $t_{1}$ and $t_{2}$, we suppose $\left\langle\mathbf{v}\left(t_{1}\right) \cdot \mathbf{v}\left(t_{2}\right)\right\rangle=1$ if the $t_{1}$ and $t_{2}$ instants occur during the same linear phase and $\left\langle\mathbf{v}\left(t_{1}\right) \cdot \mathbf{v}\left(t_{2}\right)\right\rangle=0$ otherwise. Using an exponential distribution of time spent for linear motion [see Fig. 2(c)] and no correlation between the direction of two successive linear phases [see Fig. 2(a) insert], the correlation function $C$ is written as:

$$
C(t)=v_{0}^{2} P_{\text {line }} e^{-t / T_{c}}
$$

where $T_{c}$ is the typical time and $v_{0}$ the averaged particle velocity in the linear phase. Under these assumptions, the MSD eventually reads

$$
\left\langle\left|\mathbf{r}(t)-\mathbf{r}_{0}\right|^{2}\right\rangle=2 v_{0}^{2} P_{\text {line }} t T_{c}\left(1-e^{-t / T_{c}}\right)
$$

which already predicts a transition from ballistic to diffusive motion for $t=T_{c}$. However, this approach does not explain the existence of an intermediate timescale range as indicated in Fig. 4(a). The discrepancy can be attributed to the existence of finite-time correlation in the erratic phases which may not be negligible and generate superdiffusive behavior at intermediate timescale. For $t$ greater than the averaged time spent in the erratic phases, the velocity correlation within the same erratic phase becomes negligible, and the MSD transits toward purely diffusive motion. The evolution of $\alpha$ as a function of the memory parameter Me is indicated in the inset of Fig. 4(a). Its value is a direct consequence of the proportion of time spent in both phases of motion and the finite-time velocity correlation in the erratic phases.

We measure $P_{\text {line }}$ and $P_{\text {erratic }}$ as the fraction of time spend in the linear and the erratic phases, respectively. Figure 4(b) shows the evolution of $P_{\text {line }}$ and $P_{\text {erratic }}$ with Me. We identify a critical memory parameter $\mathrm{Me}^{\star}=134.76 \pm 0.73$ corresponding to the onset of a bimodal dynamics, above which a Shil'nikov chaos is triggered. For $\mathrm{Me}>\mathrm{Me}^{\star}, P_{\text {erratic }}$ increases, following a scaling law $P_{\text {erratic }} \sim\left(\mathrm{Me}-\mathrm{Me}^{\star}\right)^{\beta}$ with an exponent $\beta=0.441 \pm 0.016$. In contrast the transition from the intermittent to the diffusive regime is progressive and we observe no signature of a discontinuity.

In this article, we have studied the dynamics of a particle propelled by a self-generated wave field in two dimensions. The wave field creates a time-dependent erratic environment with long temporal coherence coupled to the particle motion. The temporal damping of the waves controls a transition from a purely ballistic motion to erratic switches between ballistic and local diffusive motions. The difference of the two type of motion is found on the trajectories, on the speed variations, and in the properties of the associated wave field. The three dynamical features being correlated, a discriminant criterion could therefore be defined from any of these three features. The characteristics of this purely deterministic dynamics are set by the properties of a Shil'nikov chaos occurring at the vicinity of a saddle point interacting with a Hopf bifurcation. It is interesting to note that the multiscale feature is encoded by a simple deterministic wave memory. It is also worth noticing that this numerical experiment does not involve external noise or interactions with other particles, so the diffusive behavior of the particle results solely from the interaction with the wave memory.

As a final comment, it is worth noting that in other systems, the scalar field may be different from our wavelike medium which arises from the hydrodynamic specificities of the original experiment. Indeed, other bimodal dynamics of a particle coupled to a field have been reported experimentally, in which the field has no hydrodynamic nature. For example, the motion of Escherichia coli in a nutriment-filled medium is known to display the "run and tumble" dynamics [1,64]. In this case, the bacteria plays the role of the particle while the concentration of nutriments $c(\vec{r}, t)$ plays the role of the environment. Indeed, the bacteria consumes the nutriment and locally depletes the field $c(\vec{r}, t)$. The particle (bacteria) moves in new regions to find food, leaving in its trail regions depleted of nutriments. Therefore we have a synergy between the particle and its environment: The bacteria depletes the fluid of its nutriment (i.e., information is stored), and the bacteria avoids 
regions of low density through chemotactic dynamics (i.e., the environment drives the particle dynamics). Interestingly, the diffusion coefficient $D$ of the nutriment within the surrounding fluid can be related to the memory Me in our system since diffusion tends to blur the inhomogeneities created by the motion of the particle or bacteria. Yet, in contrast with this example, our system rely on memory to drive the chaotic dynamics instead of stochasticity. Another example can be seen in the Lévy flight-based dynamics of microglia caused by spreading depression [65], a pathology deeply affecting the synaptic activity and neurons excitability in the brain. The pathology is known to trigger a bimodal dynamics of this cell which, in return, acts on the whole tissue. If the role of microglia in the apparition of spreading depression and its symptoms has yet to be described, then a particle-environment model may shed a new light on the physical mechanisms at play in such a biological system.

\section{ACKNOWLEDGMENTS}

The authors thank V. Bacot and E. Fort for insightful discussions. This work was financially supported by the Actions de Recherches Concertées (ARC) of the Belgium WalloniaBrussels Federation under Contract No. 12-17/02. M.L., S.P., and Y.C. acknowledge the financial support of the French Agence Nationale de la Recherche, through Project No. ANR Freeflow ANR-11-BS04-0001. Computational resources have been provided by the Consortium des Équipements de Calcul Intensif (CÉCI), funded by the Fonds de la Recherche Scientifique de Belgique (F.R.S.-FNRS) Grant No. 2.5020.11. S.P. acknowledges the financial support of the Labex ENS-ICFP.

\section{APPENDIX}

The numerical simulations of walking droplets are made in $\mathrm{C} / \mathrm{C}++$ and the source code can be found in the Supplemental Materials [52]. We provide here an exhaustive description of the algorithm we used.

Simulations are made using the assumption that the vertical and the horizontal dynamics are not coupled to each other. The vertical dynamics corresponds to the completely inelastic bouncing ball. The horizontal dynamics is related to the interaction between the droplet and its wave field.

\section{Vertical dynamics}

Since the dynamics of an inelastic bouncing ball is periodic and does not show transient behavior, this part of the simulation is solved only once. The results one obtains are used to compute the horizontal dynamics. We assume that the surface vertical elevation is given by

$$
z_{s}(t)=A \sin \left(\omega_{s} t\right) .
$$

Because the droplet impacts on the surface are assumed to be inelastic, once the contact occurs, the droplet vertical speed relative to the surface is equal to zero. In other words, the droplet has the same vertical speed as the surface. As a consequence, the droplet only leaves the surface when the surface acceleration $\ddot{z}_{s}$ has a magnitude such as

$$
\ddot{z}_{s}=A \omega_{s}^{2} \geqslant g
$$

with $A$ being the forcing amplitude and $\omega_{s}$ the angular frequency of oscillation. Indeed, in such case, the surface "falls down" with an acceleration higher than the droplet one, i.e., $g$. The take-off instant $t^{\star}$ can be easily extracted, knowing that it corresponds to the first instant $\ddot{z}_{S}$ matches $g$

$$
t^{\star}=\frac{1}{\omega_{s}} \arcsin \left(\frac{g}{A \omega_{s}^{2}}\right) .
$$

Therefore, the take-off velocity and the take-off height can be computed. Because the droplet vertical speed is always given by the surface velocity when both are in contact, one has

$$
\dot{z}\left(t^{\star}\right)=A \omega_{s} \sqrt{1-\left(\frac{g}{A \omega_{s}^{2}}\right)^{2}}
$$

and

$$
z\left(t^{\star}\right)=\frac{g}{\omega_{s}^{2}} .
$$

The droplet, when leaving the surface, is only submitted to its weight, giving a trajectory and a vertical speed

$$
\begin{gathered}
z(t)=z\left(t^{\star}\right)+\dot{z}\left(t^{\star}\right)\left(t-t^{\star}\right)-\frac{g}{2}\left(t-t^{\star}\right)^{2}, \\
\dot{z}(t)=\dot{z}\left(t^{\star}\right)-g\left(t-t^{\star}\right) .
\end{gathered}
$$

The landing instant $t^{+}$is found by assuming $z\left(t^{+}\right)=z_{s}\left(t^{+}\right)$. Numerically, the solution is obtained via the bisection method with a relative precision of $10^{-6}$. Once $t^{+}$has been obtained, the landing velocity $\dot{z}\left(t^{+}\right)$can be computed.

The knowledge of both $t^{\star}$ and $t^{+}$defines two distinct parts of the motion. During $\Delta t_{\text {fly }}=t^{+}-t^{\star}$ ( $t_{1}$ in the main text), the droplet is assumed to have a parabolic trajectory. During $\Delta t_{\text {surf }}=4 \pi / \omega_{s}-t^{+}+t^{\star}\left(t_{2}\right.$ in the main text), the droplet is in contact with the surface and interacts with its own wave field. Knowing $\dot{z}\left(t^{+}\right)$gives a measure of the amplitude of the standing waves on the surface.

\section{Horizontal dynamics}

This part of the dynamics is solved by an event-driven algorithm. Only the two instants $t^{\star}$ and $t^{+}$are considered, since one knows the dynamics of the droplet between each.

\section{a. Parabolic trajectory}

Between $t^{+}$and $t^{\star}$, the only forces acting on the droplet are the external forces, since no contact with the surface occurs. As a consequence, the motion along the $x$ and $y$ directions is given by

$$
\begin{aligned}
& \Delta x_{\text {fly }}=\dot{x}\left(t^{\star}\right) \Delta t_{\text {fly }}, \\
& \Delta y_{\text {fly }}=\dot{y}\left(t^{\star}\right) \Delta t_{\text {fly }},
\end{aligned}
$$

where $\Delta x_{\text {fly }}=x\left(t^{+}\right)-x\left(t^{\star}\right)$ [respectively, $\Delta y_{\text {fly }}=y\left(t^{+}\right)-$ $\left.y\left(t^{\star}\right)\right]$ is the distance travelled between the impact point and the take-off point along the $x$ direction (respectively, along the $y$ direction). The impact point $\left(x\left(t^{+}\right), y\left(t^{+}\right)\right)$is stored in a memory array in order to compute the total wave field on the surface. Note that trajectories presented in this article are realized considering the impact points. 


\section{b. Interaction with the surface}

At impact, i.e., at $t=t^{+}$, the droplet loses part of its velocity because of the inelastic interaction with the interface. The vector locally perpendicular to the wave field is computed as follow. Knowing the position of all previous impact, the wave-field gradient $\nabla \zeta(\vec{r})\left(t^{+}\right)$is computed. The wave field is written as

$$
\begin{aligned}
\zeta\left(\vec{r}, t^{+}\right)= & \zeta_{0} \sum_{n=1}^{5 \mathrm{Me}+1} J_{0}\left[2 \pi \frac{\left|\vec{r}\left(t^{+}\right)-\vec{r}_{n}\right|}{\lambda}\right] \exp \left[-\frac{\left|\vec{r}\left(t^{+}\right)-\vec{r}_{n}\right|}{\delta}\right] \\
& \times \exp \left(-\frac{t^{+}-n \tau_{F}}{\operatorname{Me} \tau_{F}}\right) .
\end{aligned}
$$

The Bessel function $J_{0}$ as well as the Bessel function $J_{1}$ discussed below are computed thanks to the algorithm found in the numerical recipes. In this equation, the sum over the previous impacts is truncated for practical purpose. Indeed, since the wave amplitude is multiplied by $\exp \left[-\left(t^{+}-\right.\right.$ $\left.\left.n \tau_{F}\right)\right] / \mathrm{Me} \tau_{F}$, the last sources are negligible compared to the first ones. We choose to truncate the series at the $5 \mathrm{Me}+1$ th term since $\exp (-5)=6.7 \times 10^{-3} \ll 1$. The wave amplitude $\zeta_{0}$ is calibrated via energetic arguments. Assuming that the droplet relative kinetic energy is converted into potential energy for elevating the fluid to the height $\zeta_{0}$ leads to

$$
\zeta_{0}=\epsilon\left[\dot{z}\left(t^{+}\right)-A \omega_{s} \cos \left(\omega_{s} t^{+}\right)\right]^{2} \cos \left(\omega_{s} t^{+}\right)^{2} .
$$

The third factor in the right-hand side of this equation accounts for a correction to the amplitude because of the phase of the surface oscillation at impacts. The gradient of the field is written

$$
\begin{aligned}
\frac{\nabla \zeta\left(\vec{r}, t^{+}\right)}{\zeta_{0}}= & \sum_{n=1}^{5 \mathrm{Me}+1} \exp \left[-\frac{t^{+}-n \tau_{F}}{\operatorname{Me} \tau_{F}}-\frac{\left|\vec{r}\left(t^{+}\right)-\vec{r}_{n}\right|}{\delta}\right] \\
& \times\left\{\frac{2 \pi}{\lambda} J_{1}\left[2 \pi \frac{\left|\vec{r}\left(t^{+}\right)-\vec{r}_{n}\right|}{\lambda}\right]\right. \\
& \left.+\frac{1}{\delta} J_{0}\left[2 \pi \frac{\left|\vec{r}\left(t^{+}\right)-\vec{r}_{n}\right|}{\lambda}\right]\right\} \frac{\vec{r}\left(t^{+}\right)-\vec{r}_{n}}{\left|\vec{r}\left(t^{+}\right)-\vec{r}_{n}\right|} .
\end{aligned}
$$

Finally, the normal vector $\vec{n}\left(\vec{r}, t^{+}\right)$writes as

$$
\vec{n}\left(\vec{r}, t^{+}\right)=\left(-\partial_{x} \zeta\left(\vec{r}, t^{+}\right),-\partial_{y} \zeta\left(\vec{r}, t^{+}\right), 1\right) .
$$

Since the amplitude $\zeta_{0}$ is small compared to the wavelength $\lambda$, this expression verifies the condition $|\vec{n}|=1+\mathcal{O}\left(\zeta_{0} / \lambda\right)$. We seek for the vector $\vec{p}\left(\vec{r}, t^{+}\right)=C_{1} \vec{n}\left(\vec{r}, t^{+}\right)+C_{2}\left[\dot{\vec{r}}\left(t^{+}\right)-\right.$ $\left.\dot{z}_{s}\left(t^{+}\right) \vec{e}_{z}\right]$, perpendicular to $\vec{n}\left(\vec{r}, t^{+}\right)$and unitary. This vector lies in the plane formed by $\vec{n}\left(\vec{r}, t^{+}\right)$and $\left[\dot{\vec{r}}\left(t^{+}\right)-\dot{z}_{s}\left(t^{+}\right) \vec{e}_{z}\right]$. Mathematically, those conditions write

$$
\begin{aligned}
& \vec{p}\left(\vec{r}, t^{+}\right) \cdot \vec{n}\left(\vec{r}, t^{+}\right)=0, \\
& \vec{p}\left(\vec{r}, t^{+}\right) \cdot \vec{p}\left(\vec{r}, t^{+}\right)=1 .
\end{aligned}
$$

TABLE I. List of the parameters used in the numerical simulations.

\begin{tabular}{lr}
\hline \hline Parameter & Value (Unit) \\
\hline Forcing amplitude $A$ & $160(\mu \mathrm{m})$ \\
Forcing frequency $f_{s}$ & $80(\mathrm{~Hz})$ \\
Forcing angular frequency $\omega_{s}$ & $502.655(\mathrm{rad} / \mathrm{s})$ \\
Dimensionless acceleration $\Gamma$ & $4.12(-)$ \\
Faraday wavelength $\lambda$ & $4.75(\mathrm{~mm})$ \\
Faraday frequency $f_{F}$ & $40(\mathrm{~Hz})$ \\
Faraday angular frequency $\omega_{F}$ & $251.327(\mathrm{rad} / \mathrm{s})$ \\
Spatial damping $\delta$ & $2.5(\lambda)$ \\
Restitution coefficient $\epsilon$ & $0.006(-)$ \\
Damping time $T_{V}$ & $45(\mathrm{~ms})$ \\
Time above surface $\Delta t_{\text {fly }}$ & $14.36(\mathrm{~ms})$ \\
Time on surface $\Delta t_{\text {surf }}$ & $10.64(\mathrm{~ms})$ \\
\hline \hline
\end{tabular}

Since the impact of the droplet with the surface is assumed to be completely inelastic, the relative velocity along the normal vector $\vec{n}\left(\vec{r}, t^{+}\right)$is reduced to zero. As a consequence, the relative speed of the droplet is

$$
v_{\text {surf }}\left(t^{+}\right)=\left[\dot{\vec{r}}\left(t^{+}\right)-\dot{z}_{s}\left(t^{+}\right) \vec{e}_{z}\right] \cdot \vec{p}\left(\vec{r}, t^{+}\right),
$$

and this velocity is oriented along $\vec{p}\left(\vec{r}, t^{+}\right)$.

Between the instant $t^{+}$and $4 \pi / \omega_{s}+t^{\star}=t^{+}+\Delta t_{\text {surf }}$, i.e., the new take-off instant, the droplet is submitted to a lubrication force. This force is assumed to decrease exponentially the speed with

$$
v_{\text {surf }}\left(4 \pi / \omega_{s}+t^{\star}\right)=v_{\text {surf }}\left(t^{+}\right) \exp \left(-\frac{\Delta t_{\text {surf }}}{T_{V}}\right),
$$

where $T_{v}$ is the damping time. The distance travelled on the surface between those two instants is obtained by time integration of the previous equation

$$
d_{\text {surf }}=v_{\text {surf }}\left(t^{+}\right) T_{V}\left[1-\exp \left(-\frac{\Delta t_{\text {surf }}}{T_{V}}\right)\right] .
$$

Therefore, one has

$$
\begin{gathered}
\Delta x_{\text {surf }}=d_{\text {surf }}\left[\vec{p}\left(\vec{r}, t^{+}\right) \cdot \vec{e}_{x}\right], \\
\Delta y_{\text {surf }}=d_{\text {surf }}\left[\vec{p}\left(\vec{r}, t^{+}\right) \cdot \vec{e}_{y}\right], \\
\dot{x}\left(4 \pi / \omega_{s}+t^{\star}\right)=v_{\text {surf }}\left(4 \pi / \omega_{s}+t^{\star}\right)\left[\vec{p}\left(\vec{r}, t^{+}\right) \cdot \vec{e}_{x}\right], \\
\dot{y}\left(4 \pi / \omega_{s}+t^{\star}\right)=v_{\text {surf }}\left(4 \pi / \omega_{s}+t^{\star}\right)\left[\vec{p}\left(\vec{r}, t^{+}\right) \cdot \vec{e}_{y}\right],
\end{gathered}
$$

where $\quad \Delta x_{\text {surf }}=x\left(4 \pi / \omega_{s}+t^{\star}\right)-x\left(t^{+}\right)$and $\Delta y_{\text {surf }}=$ $y\left(4 \pi / \omega_{s}+t^{\star}\right)-y\left(t^{+}\right)$. The new position $\left(x\left(4 \pi / \omega_{s}+\right.\right.$ $\left.\left.t^{\star}\right), y\left(4 \pi / \omega_{s}+t^{\star}\right)\right)$ and the new velocity $\left(\dot{x}\left(4 \pi / \omega_{s}+\right.\right.$ $\left.\left.t^{\star}\right), \dot{y}\left(4 \pi / \omega_{s}+t^{\star}\right)\right)$ are used for a new "parabolic trajectory" phase. This algorithm is repeated until a given number of iterations is realized. Values used for the algorithm can be found in Table I.
[1] H. C. Berg and D. A. Brown, Nature 239, 500 (1972).

[2] R. Strauss and J. Pichler, J. Comp. Physiol. A 182, 411 (1998).
[3] K. Neuser, T. Triphan, M. Mronz, B. Poeck, and R. Strauss, Nature 453, 1244 (2008). 
[4] C. M. Bergman, J. A. Shaefer, and S. N. Luttich, Oecologia 123, 364 (2000).

[5] J. A. Wiens, Annu. Rev. Eco. Syst. 7, 81 (1976).

[6] R. L. Senft, M. B. Coughenour, D. W. Bailey, L. R. Rittenhouse, O. E. Sala, and D. M. Swift, BioScience 37, 789 (1987).

[7] O. Bénichou, C. Loverdo, M. Moreau, and R. Voituriez, Rev. Mod. Phys. 83, 81 (2011).

[8] W. J. O’Brien, B. I. Evans, and H. I. Browman, Oecologia 80, 100 (1989).

[9] G. M. Viswanathan, S. V. Buldyrev, S. Havlin, M. G. E. D. Luz, E. P. Raposo, and H. E. Stanley, Nature 401, 911 (1999).

[10] A. M. Reynolds and F. Bartumeus, J. Theor. Biol. 260, 98 (2009).

[11] S. Benhamou, Ecology 88, 1962 (2007).

[12] G. H. Pyke, Methods Ecol. Evol. 6, 1 (2014).

[13] A. M. Edwards, R. A. Phillips, N. W. Watkins, M. P. Freeman, E. J. Murphy, V. Afanasyev, S. V. Buldyrev, M. G. E. D. Luz, E. P. Raposo, H. E. Stanley, and G. M. Viswanathan, Nature 449, 1044 (2007).

[14] K. Pearson, Nature 72, 294 (1905).

[15] F. Bartumeus, M. G. E. D. Luz, G. M. Viswanathan, and J. Catalan, Ecology 86, 3078 (2005).

[16] G. M. Viswanathan, M. G. E. D. Luz, E. P. Raposo, and H. E. Stanley, The Physics of Foraging (Cambridge University Press, Cambridge, UK, 2011).

[17] H. Freund and P. Grassberger, Physica A 190, 218 (1992).

[18] P. Grassberger, Physica D 10, 52 (1984).

[19] Y. Couder, S. Protiere, E. Fort, and A. Boudaoud, Nature 437, 208 (2005).

[20] J. W. M. Bush, Annu. Rev. Fluid Mech. 47, 269 (2014).

[21] M. Faraday, Philos. Trans. R. Soc. London 121, 299 (1831).

[22] A. Eddi, E. Sultan, J. Moukhtar, E. Fort, M. Rossi, and Y. Couder, J. Fluid Mech. 674, 433 (2011).

[23] S. Perrard, E. Fort, and Y. Couder, Phys. Rev. Lett. 117, 094502 (2016).

[24] A. U. Oza, R. R. Rosales, and J. W. M. Bush, J. Fluid Mech. 737, 552 (2013).

[25] P. A. Milewski, C. A. Galeano-Rios, A. Nachbin, and J. W. M. Bush, J. Fluid Mech. 778, 361 (2015).

[26] E. D. Morgan, Physiol. Entomol. 34, 1 (2009).

[27] E. Bonabeau, M. Dorigo, and G. Theraulaz, Nature 406, 39 (2000).

[28] S. Protière, A. Boudaoud, and Y. Couder, J. Fluid Mech. 554, 85 (2006).

[29] A. Eddi, E. Fort, F. Moisy, and Y. Couder, Phys. Rev. Lett. 102, 240401 (2009).

[30] A. Nachbin, P. A Milewski, and J. W. M. Bush, Phys. Rev. Fluids 2, 034801 (2017).

[31] E. Fort, A. Eddi, A. Boudaoud, J. Moukhtar, and Y. Couder, Proc. Natl. Acad. Sci. USA 107, 17515 (2010).

[32] J. Moláček and J. W. M. Bush, J. Fluid Mech. 727, 582 (2013).

[33] J. Moláček and J. W. M. Bush, J. Fluid Mech. 727, 612 (2013).

[34] A. U. Oza, D. M. Harris, R. R. Rosales, and J. W. M. Bush, J. Fluid Mech. 744, 404 (2014).
[35] S. Perrard, M. Labousse, M. Miskin, E. Fort, and Y. Couder, Nat. Commun. 5, 3219 (2014).

[36] M. Labousse, S. Perrard, Y. Couder, and E. Fort, New J. Phys. 16, 113027 (2014).

[37] B. Filoux, M. Hubert, and N. Vandewalle, Phys. Rev. E 92, 041004(R) (2015).

[38] M. Labousse, S. Perrard, Y. Couder, and E. Fort, Phys. Rev. E 94, 042224 (2016).

[39] T. Gilet, Phys. Rev. E 93, 042202 (2016).

[40] M. Durey and P. A. Milewski, J. Fluid Mech. 821, 296 (2017).

[41] M. Hubert, M. Labousse, and S. Perrard, Phys. Rev. E 95, 062607 (2017).

[42] P. J. Sáenz, T. Cristea-Platon, and J. W. M. Bush, Nat. Phys. 14, 315 (2018).

[43] L. D. Tambasco, D. M. Harris, A. U. Oza, R. R. Rosales, and J. W. M. Bush, Chaos 26, 103107 (2016).

[44] S. Perrard, M. Labousse, E. Fort, and Y. Couder, Phys. Rev. Lett. 113, 104101 (2014).

[45] S. Perrard and M. Labousse, Chaos 28, 096109 (2018).

[46] S. Perrard, Une mémoire Ondulatoire: états propres, chaos et probabilités, Ph.D. thesis, Université Paris Diderot Paris VII, 2014.

[47] M. Labousse, Etude d'une dynamique à mémoire de chemin: une expérimentation théorique, Ph.D. thesis, Université Pierre et Marie Curie UPMC Paris VI, 2014.

[48] M. Durey, P. Milewski, and J. Bush, Chaos 28, 096108 (2018).

[49] O. Wind-Willassen, J. Moláček, D. Harris, and J. Bush, Phys. Fluids 25, 082002 (2013).

[50] N. Sampara and T. Gilet, Phys. Rev. E 94, 053112 (2016).

[51] V. Bacot, S. Perrard, M. Labousse, Y. Couder, and E. Fort, Phys. Rev. Lett. 122, 104303 (2019).

[52] See Supplemental Material at http://link.aps.org/supplemental/ 10.1103/PhysRevE.100.032201 for movies illustrating dynamics and a library to run simulations.

[53] F. W. J. Olver, D. W. Lozier, R. F. Boisvert, and C. W. Clark, NIST Handbook of Mathematical Functions (Cambridge University Press, Cambridge, UK, 1991).

[54] C. P. Silva, IEEE Trans. Circ. Syst. I 40, 675 (1993).

[55] F. Argoul, A. Arneodo, and P. Richetti, Phys. Lett. A 120, 269 (1987).

[56] T. Noh, Electrochim. Acta 54, 3657 (2009).

[57] M. Bassett and J. Hudson, J. Phys. Chem. 92, 6963 (1988).

[58] S. Chakraborty and S. Danaa, Chaos 20, 023107 (2010).

[59] F. Arecchi, R. Meucci, and W. Gadomski, Phys. Rev. Lett. 58, 2205 (1987).

[60] D. Dangoisse, P. Glorieux, and D. Hennequin, Phys. Rev. A 36, 4775 (1987).

[61] P. Richetti, F. Argoul, and A. Arneodo, Phys. Rev. A 34, 726 (1986).

[62] A. Koronovskii and A. Hramov, Eur. Phys. J. B 62, 447 (2008).

[63] J. Taktikos, H. Stark, and V. Zaburdaev, PLoS One 8, e81936 (2013).

[64] H. C. Berg and E. M. Purcell, Biophys. J. 20, 193 (1977).

[65] Y. Y. Grinberg, J. G. Milton, and R. P. Kraig, PLoS One 6, e19294 (2011). 